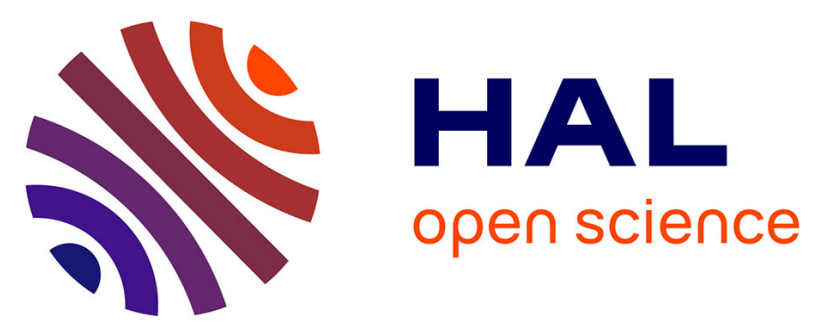

\title{
Passage of surfactant-laden and particle-laden drops through a contraction
}

Franz de Soete, Léa Delance, Nicolas Passade-Boupat, Michael Levant, Emilie Verneuil, François Lequeux, Laurence Talini

\section{- To cite this version:}

Franz de Soete, Léa Delance, Nicolas Passade-Boupat, Michael Levant, Emilie Verneuil, et al.. Passage of surfactant-laden and particle-laden drops through a contraction. Physical Review Fluids, 2021, 6 (9), 10.1103/PhysRevFluids.6.093601 . hal-03343261

\section{HAL Id: hal-03343261 \\ https://hal-espci.archives-ouvertes.fr/hal-03343261}

Submitted on 14 Sep 2021

HAL is a multi-disciplinary open access archive for the deposit and dissemination of scientific research documents, whether they are published or not. The documents may come from teaching and research institutions in France or abroad, or from public or private research centers.
L'archive ouverte pluridisciplinaire HAL, est destinée au dépôt et à la diffusion de documents scientifiques de niveau recherche, publiés ou non, émanant des établissements d'enseignement et de recherche français ou étrangers, des laboratoires publics ou privés. 


\section{Passage of surfactant-laden and particle-laden drops through a contraction}

Franz De Soete, ${ }^{1,2}$, Léa Delance, ${ }^{1,2}$ Nicolas Passade-Boupat, ${ }^{2,3}$ Michael Levant, ${ }^{2,3}$ Emilie Verneuil, ${ }^{1,2}$ François Lequeux, ${ }^{1,2}$ and Laurence Talini ${ }^{4 *}$

1 CNRS, Sciences et Ingénierie de la Matière Molle, ESPCI Paris, PSL Research University, Sorbonne Université, 75005 Paris, France.

2 Laboratoire Physico-Chimie des Interfaces Complexes, ESPCI, 10 rue Vauquelin, 75005 Paris, Bâtiment CHEMSTARTUP, Route Départementale 817, 64170 Lacq, France.

3 Total S.A. 64170 Lacq, France.

4 CNRS, Surface du Verre et Interfaces, Saint-Gobain, 93300 Aubervilliers,France

*e-mail: laurence.talini@espci.fr

Abstract: We report on the experimental behavior of oil drops suspended in water and passing in a constricted capillary tube under an imposed pressure gradient. The surfaces of droplets are covered either by colloidal solid particles or soluble surfactants. We investigate the coupling between the flow behavior and the concentration gradient in adsorbed species that are induced by surface expansion, when a water lubrication film persists between the drop and the capillary walls. For both particle-laden and surfactantladen drops, we evidence the formation of strong concentrations gradients resulting in surface tension gradients. We show how local values of surface tension can be monitored using flowrate measurements. In the case of particle-laden drops, we demonstrate the surface tension gradient is balanced by viscous friction in the lubrication film. In the case of surfactant-laden drops, we suggest a Marangoni flow opposes the decrease of surfactant concentration at the front of the drop, up to a threshold value of the surface expansion rate. Finally, we discuss how these effects increase the passage time of surfactant-laden drops in the constriction.

\section{Introduction}

Practical situations in which soft objects suspended in a liquid pass through narrow pores are ubiquitous: crude oil emulsions in porous rocks, red blood cells in constricted arteries, droplets or capsules in lab-ona-chip microfluidic devices ... The behavior of a single drop or bubble flowing through a constricted capillary has been studied both theoretically ${ }^{1}$, and experimentally ${ }^{2,3}$ : It may clog the pore, break-up, or pass through it as a whole depending on the size ratio between the drop and the pore, on the capillary number that compares viscous and capillary effects ${ }^{4}$, on the viscosity ratio of the fluid phases ${ }^{5}$ as well as on the wetting angle of the continuous liquid phase with the pore material ${ }^{6}$ and the geometrical parameters of the constriction ${ }^{7}$. The condition for clogging a constriction under an imposed pressure gradient has been thoroughly investigated for drops and bubbles ${ }^{8}$; the clogging threshold depends on the Laplace pressures across the deformed drop. More precisely, the pressure variation across the curved interface at the front of the drop opposes the driving pressure gradient whereas, at the back of the drop, the Laplace pressure 
contributes to push the drop forward. The imposed pressure threshold for clogging a constriction is therefore given by the difference between the Laplace pressures at the front and at the back of the drop.

The presence of adsorbed species at the surface of drops or bubbles changing the interfacial tension is expected to modify the clogging threshold. In practical situations, adsorbed species may be surfactants or solid particles. This is of particular interest in the process of enhanced oil recovery, which consists in injecting water in an oil well in order to detach oil from the porous rocks. Injected water may contain solid particles resulting from the drilling process, as well as added surfactants. Oil droplets with their surfaces covered by surfactants and/or solid particles form and must flow in a porous media to be carried to the surface. In the literature, the case of surfactant-laden droplets in a constriction has been examined; the decrease of surface tension induces a decrease of the Laplace pressures and hence favors the passage through a constriction by lowering the clogging threshold. Constant and uniform surface concentration of surfactants are sometimes considered ${ }^{9}$, nevertheless flow-induced concentration gradients in adsorbed species can be expected since the drop surface is expanded in the contraction. Such gradients have been evidenced in numerical studies dealing with drops or bubbles laden with insoluble surfactants passing through a constriction ${ }^{10,11}$; strong concentrations gradients between the front and the back were reported. However, possibly because of the different constriction geometries that were considered, the reported directions of the gradients are opposed, the surfactant concentration being decreased at the front of the drop in one study ${ }^{10}$, and increased in the other ${ }^{11}$. Further investigation is therefore needed to fully understand the effects at stake. In addition, the development of concentration gradients in a constriction has never been studied experimentally, as far as we know.

More generally, we address the question of the possible coupling of flow in a constriction and concentration gradients with respect to the nature of the adsorbed species. The effects of induced concentration gradients are expected to differ for particle-laden and surfactant-laden interfaces; this point is illustrated in Fig.1 where the variations of interfacial tension with covering rate or interfacial concentration are schematically represented. In the case of an interface laden with soluble surfactants, the interfacial tension $\gamma$ decreases with increasing surfactant interfacial concentration $\Gamma$, until it reaches its minimum value $\gamma_{c m c}$ when the bulk concentration reaches the critical micellar concentration $(\mathrm{cmc})$. The three configurations $\mathrm{A}, \mathrm{B}$, and $\mathrm{C}$ depict respectively a bare interface, a moderately surfactant-laden interface, and a saturated interface with a bulk surfactant concentration equal to or larger than the cmc.

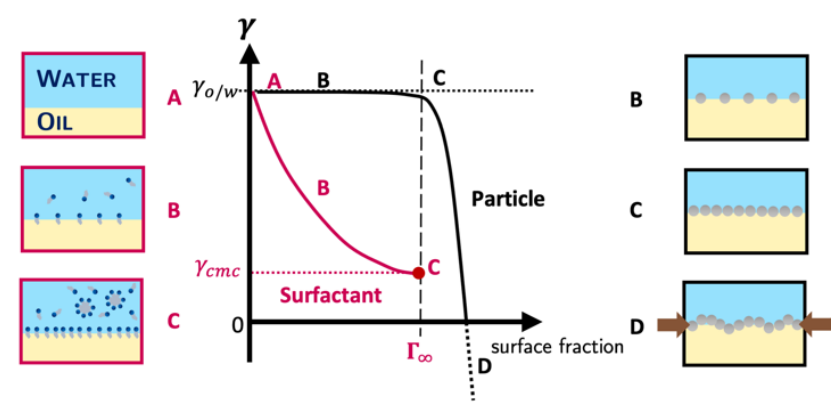

Figure 1: Schematic representation of the variation of interfacial tension $\gamma$ of particle-laden (black line) or surfactant-laden (red line) interfaces with respectively the covering rate or the interfacial concentration $\Gamma$. Schematic representations of the situations corresponding to three points of the curves are shown for surfactant (left) and particles (right) laden interfaces. 
The interfacial tension of a particle-laden interface exhibits different variations ${ }^{12}$. As long as the interface is not saturated with particles (configuration A-B) - i.e. provided the particles do not percolate - the interfacial tension $\gamma$ remains equal to the surface tension of the bare interface $\gamma_{o / w}$. Equivalently, it corresponds to a vanishing surface pressure, which is defined as $\pi=\gamma_{o / w}-\gamma$. Above particle close packing, the interfacial tension $\gamma$ decreases and conversely the surface pressure $\pi$ increases as the interface is under stress (configuration C). At large enough surface pressures (configuration D), the interface behaves as a $2 \mathrm{D}$ elastic solid and one can observe buckling, crumpling, faceting or arching depending on the particle size $^{13-15}$. The surface pressure can be larger than $\gamma_{o / w}$ and, as a consequence the surface tension can become negative, possibly resulting in buckling and/or particle expulsion ${ }^{16,17}$.

In the present work, we experimentally investigate and compare the behaviors of surfactant-laden and particle-laden drops flowing through a contraction under an imposed pressure gradient; we show that, in both cases, strong concentration gradients of either surfactants or solid particles form at the surface of the drop as a result of its expansion. We study how, in turn, these gradients modify the passage of the droplet through the contraction. We focus on drops whose surfaces are initially saturated with surfactants or particles and the varying parameter is the imposed pressure difference along the contraction.

\section{Materials and methods}

\section{A. Pressure-controlled microfluidic setup}

The experimental set-up is schematized in Fig. 2. The constricted capillaries (Hilgenberg gmbh) are of circular section and are made of borosilicate glass. We denote as $O z$ the axis of the capillary whose origin lies at the center of the constriction and, in the following, we use the cylindrical coordinates $(r, z)$. We denote as $r_{c a p}(z)$ the local radius of the capillary; far away from the constriction $r_{c a p}=600 \mu m$ and $r_{c a p}(0)=25 \mu \mathrm{m}$, the curvature radius of the constriction being $R_{c a p}=2 \mathrm{~mm}$. Two positions along the $z$ axis are defined in Fig. 2b: $z_{B}$ and $z_{F}$ are respectively the farthest upstream and downstream positions at which the tangents to the drop and to the capillary walls are the same, $z_{G}$ is the position of the mass center of the drop.

In order to impose constant pressure differences between the two ends of the capillary, it is connected to a pressure controller (OB1 MK3+ provided by Elveflow) with 0-200mbar channels dedicated to the control of pressure in the microfluidic circuit. Two low volume pressure sensors ( $0-70 \mathrm{mbar})$ placed before and after the contraction are connected to the pressure controller, allowing to regulate the pressures upstream and downstream the contraction. All junctions, unions and valves are made of PEEK with $0.5 \mathrm{~mm}$ inner diameter, and we use PTFE tubes with $0.8 \mathrm{~mm}$ inner diameter. The pressure difference $\Delta P$ that is read and controlled with the sensors corresponds to the one imposed across the contraction and was varied between 500 and $5000 \mathrm{~Pa}$. The drop deformation in the contraction is recorded using an inversed optical microscope equipped with a 55 objective and with an ultra-high speed camera (Photron) at $10000 \mathrm{fps}$, and up to 18000 fps for the fastest drops. 


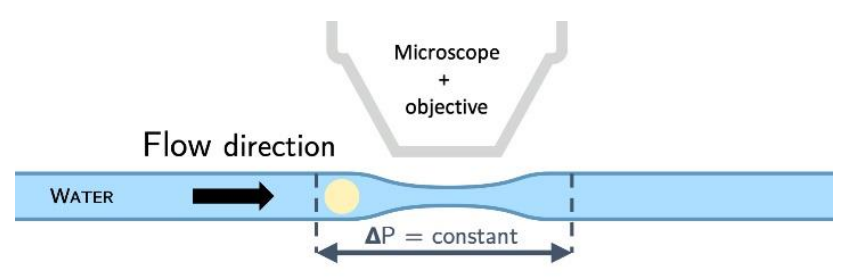

a.

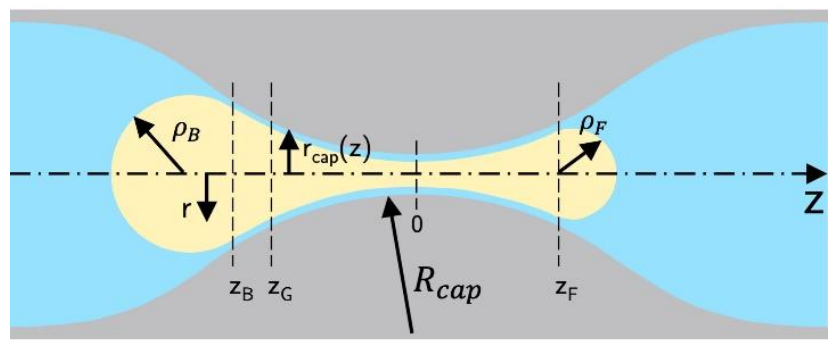

b.

Figure 2: a. Schematic representation of the experimental set-up. b. Schematic representation of an oil drop in water crossing the contraction and definition of the notations.

The experiments were performed at low capillary numbers $C a=U_{F} \eta_{w} / \gamma$, with $U_{F}$ the drop front velocity and $\eta_{w}$ the water viscosity: in all experiments $C a \leq 10^{-2}$. In addition, we consider situations in which oil does not wet the capillary walls. Consequently, there is always a lubrication water film between the drop and the capillary wall and its thickness is computed in the following. The lubrication film is always thin enough to neglect the water flowrate compared to the drop flowrate, as justified in Appendix A.

The hydrodynamic resistance $\Psi$ was computed by making several hypotheses. First, the resistance was assumed to be given by the one of the constricted part, i.e. a region comprised between positions $Z_{u p}$ and $z_{\text {down }}$ that are chosen respectively as the upstream and downstream positions along the z-axis closest to the constriction and at which $r_{c a p}=600 \mu \mathrm{m}$. Second, the flow in this part was assumed to be a HagenPoiseuille flow and the curvature of the walls was neglected. Third, in the part occupied by the drop, the contribution to flow of the lubrication water film was neglected, as mentioned above. The hydrodynamic resistance is obtained by extending the well-known relation between flowrate and pressure gradient in a circular channel ${ }^{18}$ to a varying-radius channel:

$$
\Psi=\frac{4 \eta_{w}}{\pi}\left[\int_{z_{u p}}^{z_{B}} \frac{1}{r(z)^{4}} d z+\frac{\eta_{o}}{\eta_{w}} \int_{z_{B}}^{z_{F}} \frac{1}{r(z)^{4}} d z+\int_{z_{F}}^{z_{\text {down }}} \frac{1}{r(z)^{4}} d z\right]
$$

where $\eta_{w}$ is the water viscosity, and $\eta_{o}$ the oil viscosity. In practice, we have found $\Psi$ depends weakly on When only water flows in the capillary (corresponding to the condition $z_{B}=z_{F}$ ), the hydrodynamic resistance is measured to be $\Psi=(1.4 \pm 0.1) \times 10^{12} \mathrm{~Pa} . \mathrm{s} . \mathrm{m}^{-3}$.

The drop flowrate $Q$ is measured by image analysis. Within the experimental conditions, both front and back parts of the drop can be approximated by spherical caps. By detecting the front and back extreme positions of the drop and the capillary walls we are able to deduce the front and back curvature radii, respectively $\rho_{F}$ and $\rho_{B}$, the mass center position $z_{G}$ (the zero reference is taken at the contraction center) and the drop flowrate $Q$ at any time (see Fig. 2). Figure 3 shows an example of measured time variations of the curvature radii and flow rate. In the case of particle-laden drops, the velocity at the oil/water interface 
$U_{\text {int }}$ is measured by particle tracking. The flowrate minimum is reached when the front of the drop is at the center of the contraction, which also corresponds to a minimum of the front curvature radius.

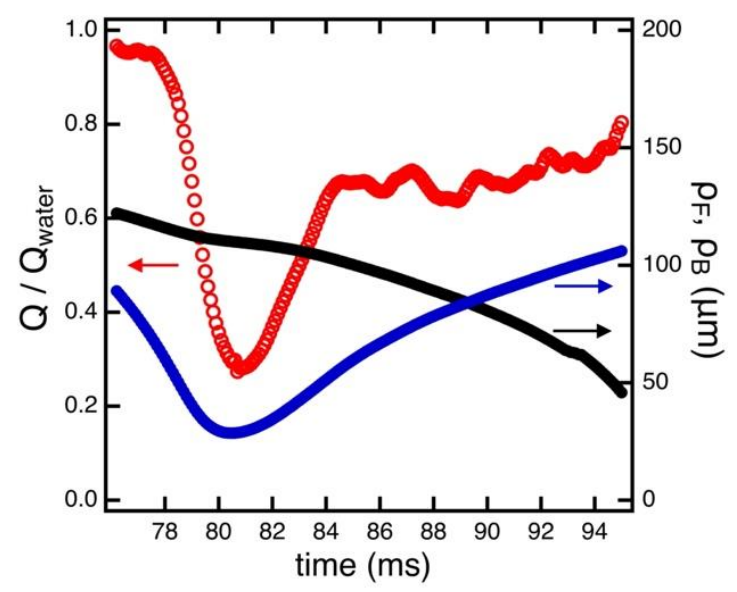

Figure 3: left axis: Normalized drop flowrate (red circles). Right axis: curvature radius of the drop back (black circles) and front (blue circles) as a function of time for a $125 \mu \mathrm{m}$ radius surfactant-laden drop at an imposed pressure difference $\Delta P=1202 P a$.

\section{B. Generation of surfactant laden drops}

The surfactant-laden oil drops (average radius $125 \mu \mathrm{m}$ ) are generated using a flow focusing device made of a tapered micropipette inserted in a squared section glass capillary, connected to the pressure controller. Oil flows in the tapered micropipette, and surfactant solution is the outer phase. The difference between the oil and water pressures controls the size and number of oil in water drops, and their generation frequency. The surfactant solution is made of deionized water with $10^{-4} \mathrm{~mol} . \mathrm{L}^{-1}$ added $\mathrm{NaCl}$ and CTAB (cetyltrimethylammonium bromide). CTAB critical micellar concentration (cmc) is $9 \times 10^{-4} \mathrm{~mol}^{-1}$ and its concentration in the solution is either 1,5 or 10 times $\mathrm{cmc}$. The oil used for drop generation is $n$-dodecane from VWR, of viscosity $1.3 \times 10^{-3} \mathrm{~Pa}$. .

The relation between surface concentration of surfactant, $\Gamma$, and surface tension $\gamma$ is well described by:

$$
\gamma=\gamma_{o / w}+2 R T \Gamma_{\infty} \log \left(1-\frac{\Gamma}{\Gamma_{\infty}}\right)
$$

where $R$ is the ideal gas constant, $T=298 \mathrm{~K}$ is the temperature at which all the experiments are performed and Log stands for the Napierian logarithm. $\Gamma_{\infty}$ is the surface concentration at saturation and was measured to be $2.5 \times 10^{-6} \mathrm{~mol} . \mathrm{m}^{-2}$, in agreement with existing data on CTAB at oil/water interface ${ }^{19-21}$.

The interfacial tension of oil with water was measured using a pendant drop experiment (Teclis), and was found to be $38.6 \pm 0.6 \mathrm{mN} . \mathrm{m}^{-1}$ at zero CTAB concentration and $5.6 \pm 0.6 \mathrm{mN} . \mathrm{m}^{-1}$ at concentrations larger than $\mathrm{cmc}$.

\section{Preparation of Pickering emulsions}


Same $\mathrm{NaCl}$ aqueous solution and oil have been used for the Pickering emulsion formulation as for the surfactant-stabilized emulsions. The stabilizing particles are monodisperse silica microspheres of $1.5 \mu \mathrm{m}$ diameter (Fiber Optic Center) that are highly hydrophylic.

The particles are first dispersed in $10 \mathrm{~mL} \mathrm{NaCl}$ solution using an ultrasonic probe $(20000 \mathrm{~Hz}, 40 \%$ of maximum intensity). Oil is further added and the emulsion is obtained by mixing at $18000 \mathrm{rpm}$ during 30s. Full emulsification is ensured by the absence of neither creamed oil layer nor sedimented solid particles. The diameter of the formed drops $D_{\text {drop }}$, measured with optical microscopy, ranges from $200 \mu \mathrm{m}$ to $300 \mu \mathrm{m}$. The surface fraction $C=0.86 \pm 0.04$ of particles at the interface is measured from close views (inset of Fig. 4). As observed on microscopy images, the drops are densely covered by particles, almost at hexagonal close packing.

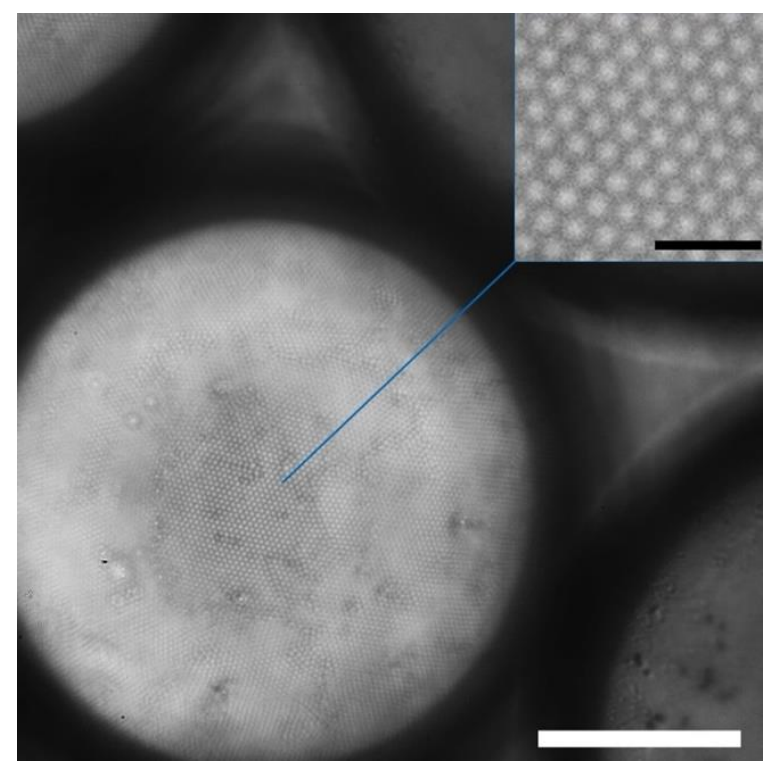

Figure 4: View of a Pickering drop covered with silica microspheres (scale-bar 50um) and close view of its surface (inset, scale bar 5 $\mu \mathrm{m}$ ). The surface fraction covered with particles was measured to be $C=$ $0.86 \pm 0.04$ from close up views.

Assuming the emulsions are in the limited coalescence regime ${ }^{22}$, the surface fraction is given by:

$$
C=\frac{D_{d r o p}}{4 d_{s}} \frac{\rho_{o} M_{S}}{\rho_{s} M_{o}}
$$

where $d_{s}=1.5 \mu \mathrm{m}$ is the silica particle diameter. The index o and s respectively refer to oil and silica and $\rho$ and $\mathrm{M}$ respectively denote the density and total mass. With $\rho_{s}=2000 \mathrm{~kg} \cdot \mathrm{m}^{-3}, \rho_{o}=778 \mathrm{~kg} . \mathrm{m}^{-3}$, we find with eq. (3) that $C=0.86$, which matches the value measured optically, and is consistent with an emulsion generation limited by coalescence. We thus obtain a collection of Pickering drops that we dilute so that they can be pushed one by one in the constricted capillary tube.

\section{Results}

\section{A. General features}


When a drop flows through the constricted capillary, at each position the relation between pressure difference and flowrate is given by ${ }^{2}$ :

$$
Q=\frac{1}{\psi}\left(\Delta P+\frac{2 \gamma_{B}}{\rho_{B}}-\frac{2 \gamma_{F}}{\rho_{F}}\right)
$$

where $Q$ is the drop flowrate measured by image analysis and $\Psi$ the hydrodynamic resistance given by eq. (1). The indexes B and F respectively refer to the back and the front of the drop and $\gamma$ and $\rho$ respectively denote the surface tension and radius of curvature. The Laplace pressure resulting from the curvature of the capillary of radius $R_{\text {cap }}$ (see Fig. 2b) is neglected.

Both the imposed pressure and Laplace pressure at the back contribute to push the drop forward in the contraction and appear with a positive sign in eq. (4). In contrast, the Laplace pressure at the front opposes forward motion and decreases the flowrate. The minimum curvature radius of the drop front is reached at the center of the contraction, i.e. at position $z=0$. In addition, it is about 4 times smaller than the curvature radius at the back. As a result, at this position, the pressure due to the curvature at the back is small and the flowrate is expected to be minimum (see Fig. 3). Therefore, the pressure threshold for clogging corresponds to cases where the flow rate becomes zero at the center of the contraction.

\section{B. Flow of a Pickering drop through the contraction}

In Fig. 5a are displayed different photographs of a particle-laden drop crossing the contraction together with the variations of flowrate as a function of the position of its mass center, $z_{G}$. Photograph (i) shows the drop entering the contraction. The drop surface further expands up to a maximum corresponding to the situation of photograph (iii) for which the mass center of the drop is at the center of the contraction, i.e. $z_{G}=0$. As previously mentioned, the minimum value for flowrate is reached when the front of the drop is at the contraction center (photograph ii), and must be non-zero for the drop to pass through.

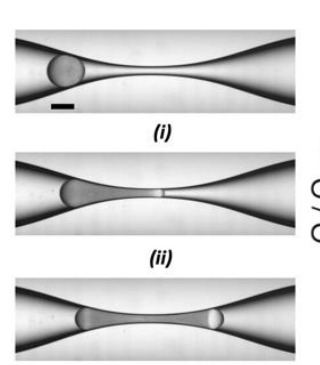

(iii)

a.

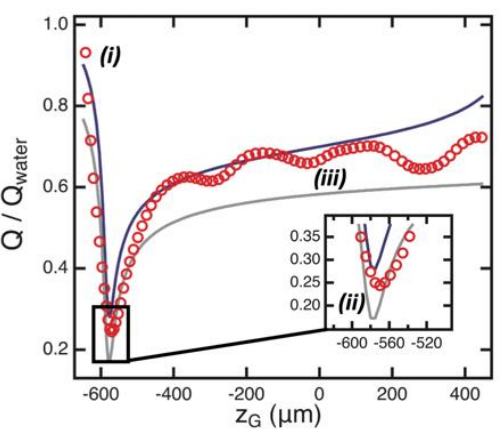

b.

Figure 5: Particle-laden drops. a. Views of the key positions of the drop in the contraction. (i) Drop entering

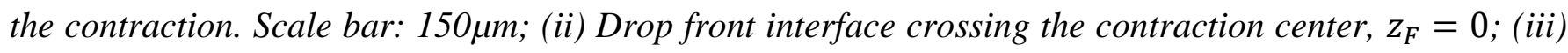
Drop mass center in the middle of the contraction, $z_{G}=0 . b$. Drop normalized flowrate as a function of the drop mass center position for a particle-laden drop. Symbols refer to experimental data, blue full line to computed flowrate with $\gamma_{B}=\gamma_{F}=\gamma_{o}$; ; grey full line to the computed flowrate with $\gamma_{B}=0$ and $\gamma_{F}=$ $\gamma_{o / w}$. The inset is a zoom at the minimum of flowrate corresponding to photograph (ii). The drop radius is $136 \mu \mathrm{m}$ and the pressure difference $4365 \pm 20 \mathrm{~Pa}$. 
The difference of gray shades between the front apex (white) and the rest of the drop (gray) on photograph (ii) indicates that a bare oil-water interface forms at the drop front. Photograph (iii) shows that this interface remains bare at least until $z_{G}=0$. As emphasized above, the drop surface is initially almost saturated with particles. As soon as the drop enters the contraction, its surface expands resulting in a decrease of the particles density at the interface. Referring to the variations of interfacial tension schematized in Fig. 1, all these observations indicate that the front part of the drop is exploring situations B to A. Thus, we assume that the front interfacial tension is equal to the interfacial tension of a bare oil-water interface $\gamma_{\mathrm{F}}=\gamma_{\mathrm{o} / \mathrm{w}}$ during the whole experiment. In contrast, particles concentrate at the back of the drop, and the interfacial tension $\gamma_{\mathrm{B}}$ is expected to become smaller than $\gamma_{\mathrm{o} / \mathrm{w}}$ corresponding to an evolution from situation $\mathrm{B}$ to situation $\mathrm{C}$ or D in Fig. 1.

Figure 5.b. shows the measured drop flowrate $Q$ as a function of the position of the droplet mass center. The flowrate is normalized by the one without droplet (i.e. with water only) at the same pressure difference $\Delta P$. Here again, the minimum flowrate is observed when the front of the drop is at the center of the contraction, corresponding to the case of photograph (ii). The solid lines show the predicted flowrates computed using eq. (4). All quantities appearing in eq. (4) are measured, except interfacial tensions. From the observed bare drop front, we infer that $\gamma_{\mathrm{F}}=\gamma_{\mathrm{o} / \mathrm{w}}$. The values of the back interfacial tension is not known a priori since the interface enriches with particle, and the two curves correspond respectively to its possible lower and upper values : $\gamma_{\mathrm{B}}=0$ and $\gamma_{\mathrm{B}}=\gamma_{\mathrm{o} / \mathrm{w}}$. The first value corresponds to a maximal particle density at the back and is associated with the onset of buckling (situation C-D of Fig.1). In contrast, a constant interfacial tension $\gamma_{\mathrm{o} / \mathrm{w}}$ is expected if the density remains smaller than its saturation value (situation B of Fig.1). As evidenced in Fig. 5a, the experimental curve lies between these two computed curves, whatever the droplet position. Therefore we infer that $0<\gamma_{\mathrm{B}}<\gamma_{\mathrm{o} / \mathrm{w}}$ at all positions, and in particular at the one corresponding to minimum flowrate.

\section{Flow of a surfactant laden drop through the contraction}

We now turn to the experiments performed with surfactant-laden drops. Figure 6.a. presents different photographs of a surfactant-laden drop crossing the contraction. Photographs (i), (ii), and (iii) show the same situations as in the case of a particle-laden drop previously presented. In contrast to particle-laden drops, the surfactants cannot be visualized, hence their surface concentration cannot be inferred from the images. Yet, at the back, the concentration is not expected to increase since it is initially saturated, therefore $\gamma_{B}=\gamma_{c m c}$. At the front, similarly to particle-laden interfaces, we expect either a constant or decreasing surface concentration which results, this time, in an increase of the front interfacial tension, i.e. $\gamma_{c m c}<$ $\gamma_{F}<\gamma_{o / w}$. 


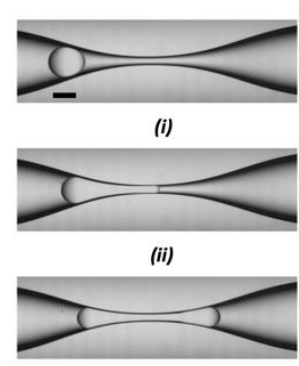

(iii)

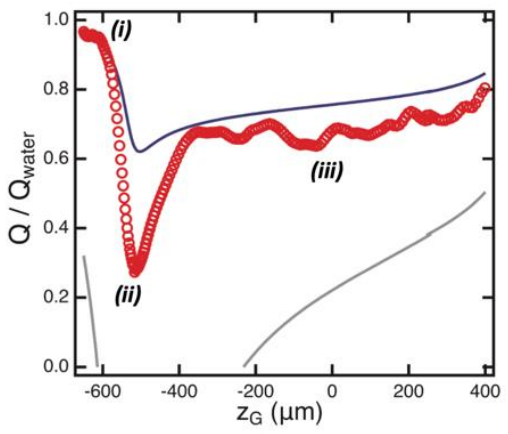

b.

Figure 6: a. Photographs of the key positions of a surfactant-laden drop at $5 \mathrm{cmc}$. (i) Drop entering the contraction. Scale bar: $150 \mu m$; (ii) Drop front interface crossing the contraction center, $z_{F}=0$; (iii) Drop mass center in the middle of the contraction, $z_{G}=0 . b$. Drop normalized flowrate as a function of the drop mass center position for a surfactant-laden drop. Symbols refer to experimental data, blue full line to computed flowrate with $\gamma_{B}=\gamma_{F}=\gamma_{c m c}$; grey full line to the computed flowrate with $\gamma_{B}=\gamma_{c m c}$ and $\gamma_{F}=$ $\gamma_{o / w}$. The drop radius is $125 \mu \mathrm{m}$ and the pressure difference $\Delta P=1202 \pm 20 \mathrm{~Pa}$.

The measured drop flowrate $Q$ normalized by $Q_{w a t e r}$ as a function of $z_{G}$ is shown in fig. $6 \mathrm{~b}$. In this case as well, the minimum value for flowrate is reached when the front is at the center of the contraction. The blue and gray lines were computed using eq. (4) with respectively $\gamma_{F}=\gamma_{c m c}$ and $\gamma_{F}=\gamma_{o / w}$. In both cases $\gamma_{B}=$ $\gamma_{c m c}$. The experimental data lies between these two extreme situations, confirming the concentration at the front does not remain constant but decreases. Interestingly, the experimental minimum flowrate is more than twice smaller than the model with both interfacial tensions kept constant. The induced decrease of surfactant concentration can therefore be expected to modify the passage time of the drop.

In the following, we focus on the position of the drop corresponding to the minimum flowrate, which conditions the passage of the drop. By adjusting the minimum flow rate computed with eq. (4) to the experimental one, we have determined the unknown interfacial tension in each case. More precisely, for each $\Delta P$, the back interfacial tension of particle-laden drops was computed from the value of the minimum using the relation inferred from eq. (4):

$$
\gamma_{B}=\frac{\rho_{B}}{2}\left(\Psi Q-\Delta P+\frac{2 \gamma_{\mathrm{o} / \mathrm{w}}}{\rho_{F}}\right)
$$

Similarly, the front interfacial tension of surfactant-laden drops was computed from the flow rate minimum with a back interfacial tension $\gamma_{c m c}$, i.e.:

$$
\gamma_{F}=\frac{\rho_{F}}{2}\left(\Psi Q-\Delta P-\frac{2 \gamma_{\mathrm{cmc}}}{\rho_{B}}\right)
$$

All parameters of eq. (5) and (6) are either known $\left(\Psi, \Delta P, \gamma_{\mathrm{o} / \mathrm{w}}, \gamma_{\mathrm{cmc}}\right)$ or can be measured by image analysis $\left(\rho_{B}, \rho_{F}\right.$ and $\left.Q\right)$. We have found that, at the center of the contraction, all terms of the differences involved in eq. (5) and (6) are at least of a few tenths of $\Delta P$, allowing accurate measurements of surface tensions using these equations. Actually, the main error comes from the flowrate term yielding an uncertainty of less than $20 \%$ on surface tensions. In the following section, $w$ e discuss the resulting values of surface tensions $\gamma_{B}$ and $\gamma_{F}$ as a function of $\Delta P$ and we suggest a picture of the various transport phenomena at stake. 


\section{Discussion}

We show in this section that the values of interfacial tension determined from the flowrate curves can be successfully predicted by considering the processes involved in the creation of concentration gradients of the adsorbed species. The first step consists in examining the velocity of these species, i.e. the interfacial velocity of the drop.

\section{A. Water lubrication film and interfacial velocity}

The lubrication film formed when a drop or bubble moves at constant velocity in a cylindrical capillary has been first described by Bretherton for capillary numbers $C a<10^{-3} 23$. He showed that the film thickness only depends on the capillary number and the tube radius. More recently, an empirical law was suggested to predict the film thickness at larger capillary numbers ${ }^{24}$, which was further modified on the basis of theoretical arguments ${ }^{25}$. In the present work, the radius of the capillary tube is varying across the contraction, resulting in a varying drop velocity and thus in a varying capillary number. However, considering the large value of the curvature radius of the capillary, $R_{\text {cap }}$ compared to the drop radius, these variations are small and can be neglected. Therefore, we use the expression established in ${ }^{25}$ for the thickness of the lubrication film, yielding at the center of the contraction:

$$
h=r_{c a p}(0) \frac{1.34 C a^{2 / 3}}{1+3.73 C a^{2 / 3}}
$$

In the case of particle-laden drops, the lubrication film thickness obtained from eq. (7) ranges from 0.8 to $1.5 \mu \mathrm{m}$, i.e. is always larger than the radius of the colloidal particles, $h>d_{\text {silica }} / 2$. Therefore, we consider the particles freely move at the oil-water interface since there is no solid friction between the particles and the capillary walls. For surfactant-laden drops, the film thickness obtained with eq. (7) ranges from 0.1 to $2.7 \mu \mathrm{m}$.

The velocity profiles in the drop and in the film are schematized in Fig. 7. Computation of the velocity $U_{\text {int }}$ of the o/w interface with respect to the capillary tube is detailed in Appendix A. As shown in the Appendix, neglecting the contribution to the interfacial velocity of both Marangoni effect and interfacial viscosity yields the approximation:

$$
U_{\text {int }} \approx 2 \frac{\eta_{o}}{\eta_{w}} \frac{\rho_{F}^{2} h}{\mathrm{r}_{\text {cap }}^{3}} U_{F}
$$

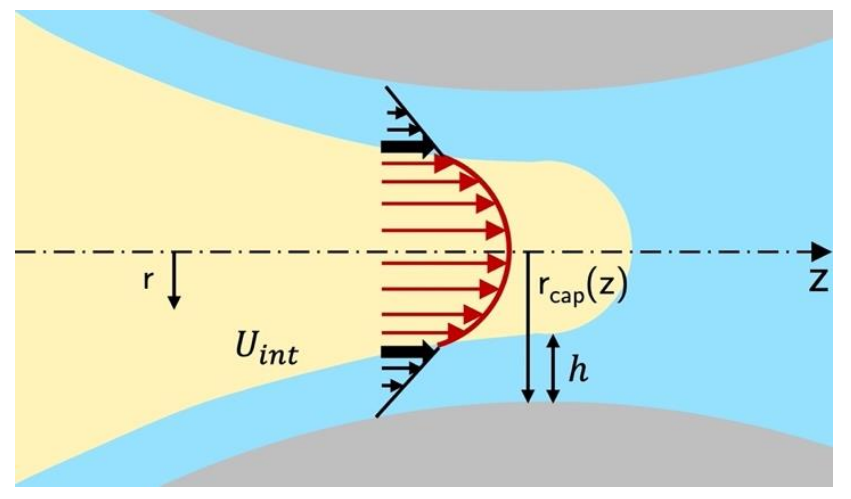


Figure 7: Schematic representation of the velocity profiles in the lubrication film and in the drop. The velocity at the drop interface is denoted $U_{\text {int }}$.

The condition $\frac{\rho_{F}^{2} h}{r_{\text {cap }}^{3}} \ll 1$ is met in all the investigated experimental conditions and the oil and water viscosities are very close. Therefore, from eq. (8), the interfacial velocity $U_{\text {int }}$ is always smaller than the front drop velocity $U_{F}$, provided Marangoni effects are negligible.

In the following, we explain how the transfers of the interfacial material (particles or surfactants) set the interfacial tensions measured at the flowrate minimum. In the case of surfactant-laden drops, we measure the front surface expansion rate $\dot{A}$ and show it is the key parameter to discuss the variations of the front interfacial tension. In the case of particle-laden drops, we demonstrate that the mechanical balance on particles allows a prediction of the back interfacial tension.

\section{B. Particle-laden drops: mechanical balance}

The different interfacial effects at stake for particle-laden drops are schematically represented in Fig. 8. In the previous section, we concluded from the experimental measurements that $\gamma_{B}<\gamma_{o / w}$, which corresponds to a non-zero surface pressure $\pi\left(z_{B}\right)=\gamma_{o / w}-\gamma_{B}>0$ at the back of the drop. In contrast, the interfacial tension at the front remains equal to the oil/water interfacial tension and the surface pressure is strictly zero: $\pi\left(z_{F}\right)=0$. Consequently, a surface pressure gradient $\left[\pi\left(z_{F}\right)-\pi\left(z_{B}\right)\right] /\left(z_{F}-z_{B}\right)$ forms along the length $\left(z_{F}-z_{B}\right)$ of the drop. This gradient is balanced by hydrodynamic friction in the lubrication film of thickness $h$, which yields:

$$
\gamma_{B} \sim \gamma_{o / w}-\eta_{w}\left(z_{F}-z_{B}\right) \frac{U_{\text {int }}}{h}
$$

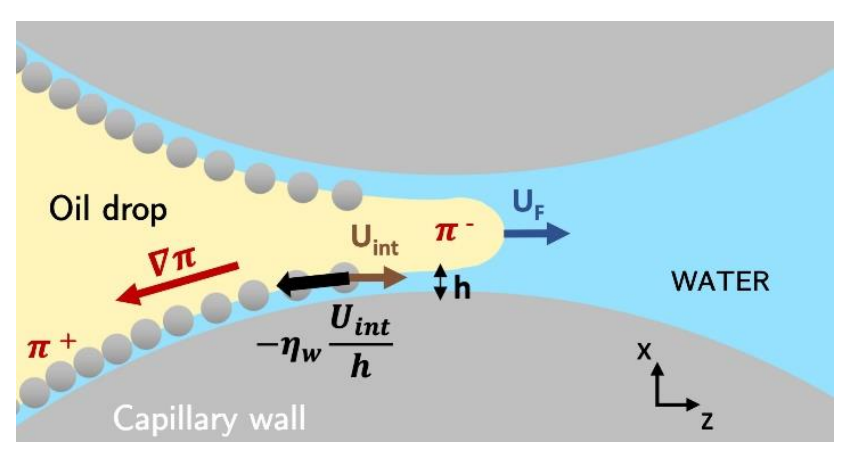

Figure 8: Schematic representation of the particle-laden interface; $\pi$ is the surface pressure ( $\pi=\sigma z z$, the surface stress in the principal direction $z), h$ the water film thickness, $U_{\text {int }}$ the interfacial velocity, $\eta_{w}$ the water viscosity. The particle density gradient generates a surface pressure gradient along the drop, which is balanced by the hydrodynamic friction in the water film.

Equation (9) provides a measurement of the surface tension at the back of particle-laden drops from the experimental values of the interface velocity, which is measured by tracking the particle front at all times. This tracking method is based on the contrast difference that can be observed on Fig. 5.a between drop areas covered with and free of particles. We have found velocities ranging from $10^{-2} \mathrm{~m} . \mathrm{s}^{-1}$ to $0.6 \mathrm{~m} . \mathrm{s}^{-1}$ always smaller than the front velocity. In Fig. 9, we compare the resulting value of the back interfacial 
tension with the one provided by flowrate measurements following eq. (5). The agreement between both measurements is excellent. The only varying parameter between each point is the imposed pressure difference across the contraction, $\Delta P$, resulting in different drop front velocities $U_{F}$. As expected, the back interfacial tension decreases with increasing imposed pressure gradient. We emphasize Fig. 9 validates the assumptions made to obtain the thickness of the lubrication film since this thickness is used to compute the ordinate values. Neglecting the variations of both the capillary radius and drop velocity is therefore justified.

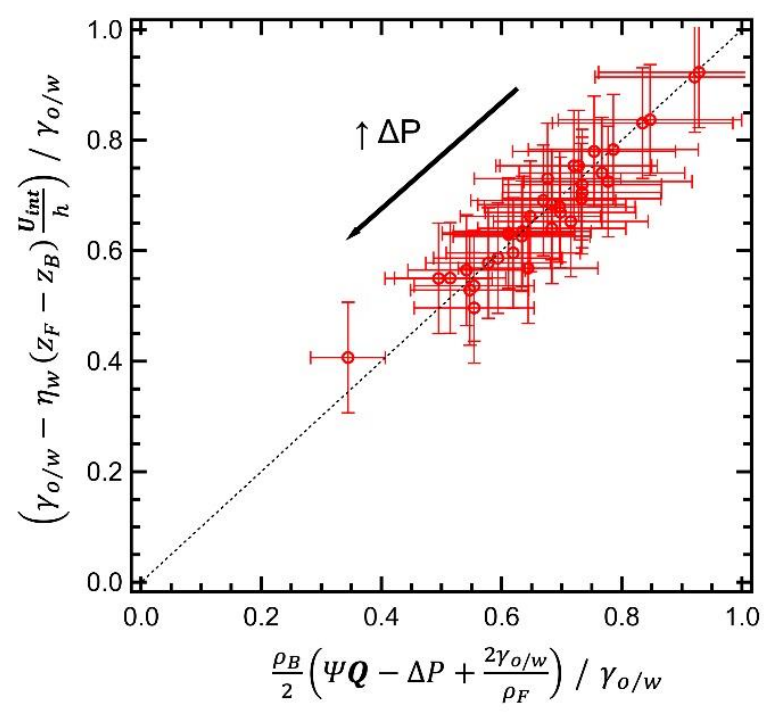

Figure 9: Interfacial tension at the back of a particle-laden drop computed with eq. (9) as a function of the one computed with eq. (5) at the minimum flowrate. Error bars result from uncertainty on interfacial velocity measurements (vertical), and flowrate measurements (horizontal). The only varying parameter is the imposed pressure difference across the contraction, $\Delta P$.

\section{Surfactant-laden drops: surfactant transfers}

We have systematically measured the front surface tension of surfactant-laden drops at the flow rate minimum using eq. (6). As expected, it depends on the imposed pressure difference, $\Delta P$. The surfactant interfacial concentration is expected to decrease as the drop surface expands: hence, at the front of the drop, for each $\Delta P$ we have measured the surface expansion rate $\dot{A}$ from image analysis. The expansion rate is defined as the relative surface variation with time and we show in Appendix B its value at the center of the contraction can be simply expressed as:

$$
\dot{A}=\frac{1}{S_{F}} \frac{d S_{F}}{d t}=\frac{U_{F}}{\rho_{F}}
$$

The measured front surface tension is shown as a function of $\dot{A}$ for different surfactant concentrations above the cmc. 


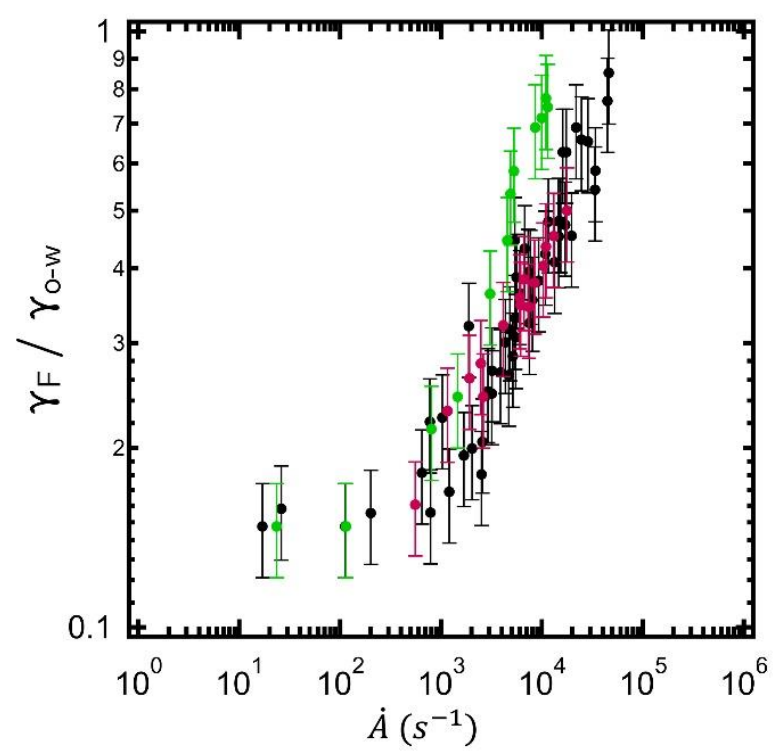

Figure 10: Front surface tension of surfactant-laden droplets normalized by the surface tension of bare oilwater interface as a function of the expansion rate of the drop at the front and for different surfactant concentrations: $1 \mathrm{cmc}$ (green circles), $5 \mathrm{cmc}$ (black circles) and $10 \mathrm{cmc}$ (red circles). Surface tension is computed following eq. (6) at the position corresponding to the minimum flowrate. The expansion rate is computed with eq. (B5) at the same position.

Strikingly, no significant influence of surfactant concentration is observed on the front surface tension. At small expansion rates, $\gamma_{F}$ is constant and equal to $\gamma_{c m c}$. Above $\dot{A} \sim 10^{3} S^{-1}$, it steeply increases and further tends toward values close to $\gamma_{o / w}$ at the largest expansion rates, evidencing a significant decrease in surfactant concentration at the front surface of the drop. The corresponding surface concentrations $\Gamma_{F} / \Gamma_{\infty}$ computed using eq. (2) are shown in Fig. 11.

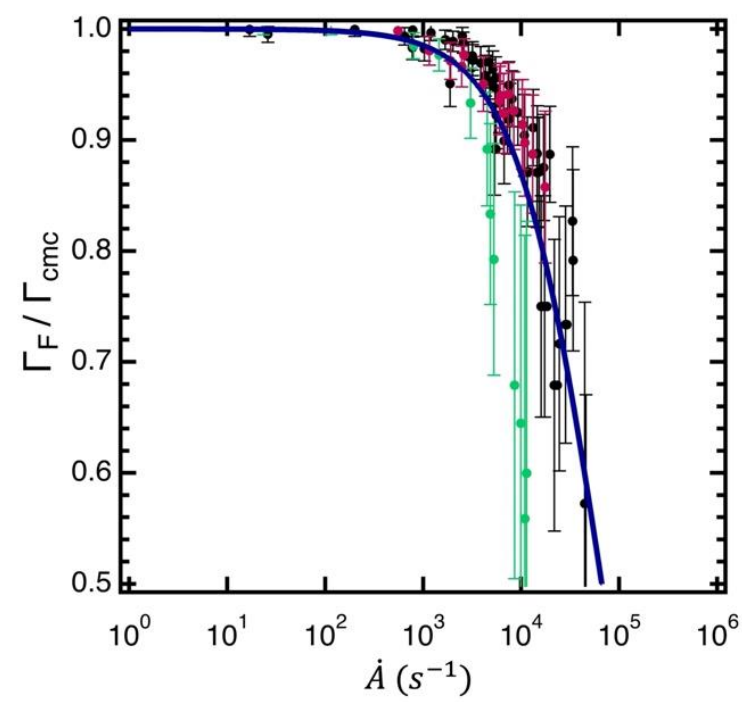

Figure 11: Surface concentration normalized by its value at the cmc computed from the data of Fig. 10 using eq. (2) for different surfactant bulk concentrations (same as Fig. 10). The solid line is a guide to the eye. 
We now discuss the different effects influencing the surface concentration of surfactants at the front of the drop. The decrease of surface concentration resulting from surface expansion is opposed by both a Marangoni interfacial flow and the feeding of surfactants from the bulk to the depleted interface. Actually, as the drop moves forward, surfactants and micelles from the bulk are driven towards the drop front by an advection/diffusion process, and further adsorb within a finite time at the $\mathrm{o} / \mathrm{w}$ interface. The limiting step of this process can either result from the time needed by surfactants to diffuse from the bulk to the interface, or from the finite adsorption time. The latter is expected for charged surfactants as CTAB ${ }^{26}$, since charge interactions induce an energy barrier for adsorption at the interface. We demonstrate in Appendix $\mathrm{C}$ that, whatever the nature of the process limiting adsorption of surfactants from the bulk, the decrease of surface concentration would occur above a threshold value of surface expansion. Consistently, a threshold value is evidenced by the data of Fig. 11. However, if diffusion was the limiting step, the threshold should depend on surfactant bulk concentration and in particular be zero at a concentration of cmc, as stated in Appendix C. Clearly, the experimental data shows no significant effect of bulk concentration on the variations of surface concentration. Similarly, if the threshold did result from a finite adsorption time, then its value would depend on surfactant concentration ${ }^{26}$. Therefore, we conclude that it is not the adsorption of surfactants from the bulk that sets the threshold value above which surface concentration decreases.

We suggest that the observed threshold rather reflects the influence of a Marangoni flow. In Appendix A, we show the Marangoni number can be of the order of unity at the smallest values of front velocity. Therefore, at these velocity values, a Marangoni flow can oppose the decrease of surface concentration. We describe this effect by considering the front velocity is decreased by the Marangoni velocity $U_{M a}$ and we define an effective value for the expansion rate in which the velocity is $U_{F}-U_{M a}$ instead of $U_{F}$ as in eq. (10). Using the Marangoni velocity derived in Appendix A yields for the effective expansion rate:

$$
\dot{A}_{e f f}=\frac{U_{F}}{\rho_{F}}\left(1-\frac{\partial \gamma}{\partial z} \frac{\rho_{F}}{\eta_{w}} \frac{1}{U_{F}^{1 / 3} U_{c}^{2 / 3}}\right)
$$

Where $U_{c}$ is the capillary velocity. Equation (11) is written at the center of the contraction at which $r_{c a p}=$ $\rho_{F}$.

At small drop velocities, the Marangoni flow can cancel the effects of surface expansion whereas it becomes negligible at large drop velocities. The crossover velocity between the two regimes corresponds to a zero effective expansion rate, i.e. to a threshold of the expansion rate:

$$
\dot{A}_{t h}=\left(\frac{1}{\eta_{w}} \frac{\partial \gamma}{\partial z}\right)^{3} \frac{\rho_{F}^{2}}{U_{c}^{2}}
$$

Using the same numerical values as in Appendix A, we find $\dot{A}_{t h} \approx 8 \times 10^{3} \mathrm{~s}^{-1}$. This threshold value is in very good agreement with the one evidenced in Fig. 11.

Above the threshold value, the decrease of the surfactant concentration results from a balance of the different effects respectively decreasing and increasing surface concentration. Its complete description should therefore take into account both the diffusion and adsorption processes, and is beyond the scope of the present work. Nevertheless, the experimental master curve describing the variations of surface 
concentration with surface expansion allows an accurate prediction of the passage of the drops, as shown in what follows.

\section{Passage time}

In light of what precedes, we now focus on the consequences of the induced concentration gradients on the passage time of a surfactant-laden drop. No significant effect is expected on the clogging threshold since concentrations gradients only appear for large enough expansion rates, i.e. not for vanishing velocities. However, for large rates resulting in large variations of the front surface tension, we expect an increase of the passage time of the drop.

We arbitrarily define a passage time as the time needed for the mass center of the drop to move from $z_{1}$ to $z_{2}$, where $z_{1}$ is the position along the z-axis at which the drop starts losing its spherical shape, and $z_{2}$ the position at which the spherical shape is recovered. The passage time can be directly measured in the experiment and, since we fully describe the surface tension variations at the surface of the drop, it can also be computed using:

$$
t_{p}=\int_{z_{1}}^{z_{2}} \frac{\pi r^{2}(z)}{Q(z)} d z
$$

Where $Q(z)$ is the drop flow rate predicted using eq. (5) in which $\gamma_{B}=\gamma_{c m c}$ and $\gamma_{F}$ is computed at each position from the expansion rate, using an empirical expression determined from the curve of Fig. 10. Since the expansion rate itself depends on the flowrate, an equation verified by $Q$ is obtained, which is further solved numerically and injected in eq. (13), itself numerically integrated.

Measurements of $t_{p}$ are shown in Fig. 12 as a function of the imposed pressure normalized by the clogging pressure, together with the numerically integrated passage time given by eq. (13). They are compared with the passage times of constant surface tension drops respectively without surfactant $\left(\gamma_{F}=\gamma_{o / w}\right)$ and with a saturated interface $\left(\gamma_{F}=\gamma_{c m c}\right)$. The pressure clogging threshold corresponds to the pressure value for which $t_{p}$ becomes infinite. As expected, the threshold is strongly decreased by the addition of surfactant, and unperturbed by considering concentration gradients effect. The experimental passage times are well described by the predicted ones, emphasizing the relevance of the simple model we have used. They are slightly larger than the passage times obtained by considering a constant surface tension and the effect is enhanced close to the clogging threshold. For instance, the time is almost twice larger for a pressure difference twice larger than the clogging threshold. Although moderate, the effect is expected to be amplified for drops passing in multiple contractions. Therefore, induced surface concentration gradients need to be considered in order to fully predict the flows of surface-laden droplets in porous media. 


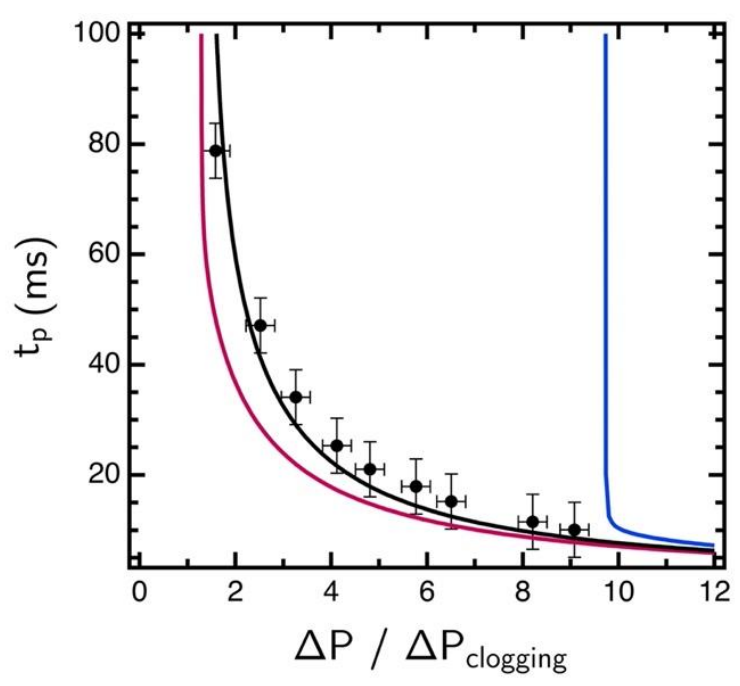

Figure 12: Passage time of a surfactant-laden drop as a function of the imposed pressure difference normalized by the clogging pressure given by eq. (5) with $Q=0$. The time is the one needed by the drop to cross the contraction, i.e. to move from position $z_{1}$ to $z_{2}$ (see Fig. 2). Experimental data (symbols) is shown with the ones predicted for a constant surface tension at the front, respectively $\gamma_{c m c}$ (red line) and $\gamma_{o w}$ (blue line). The predicted crossing time when the front surface tension is modified by the surface expansion is shown as a black line.

\section{Conclusion}

We report experiments performed with particle-laden and surfactant-laden drops passing through a contraction under an imposed pressure, resulting in a large surface expansion of the drop. We have shown that both natures of drops have a qualitatively similar behavior when crossing the contraction, with a decrease in the concentration of adsorbed species at their fronts. The resulting variations in surface tension as a function of flow rate we measure are in excellent agreement with the ones we predict. In particular, we show that above a threshold velocity value, a Marangoni flow opposes the decrease of surfactant concentration at the front interface due to surface expansion. We demonstrate the induced surface tension gradients result in larger passage times through the contraction. Our results provide the first quantitative experimental study of the concentration gradients induced by drop deformation in a constriction and sheds new light on the transport in porous media of droplets with adsorbed species at their surfaces.

\section{Appendix A: interfacial velocity}

In this appendix, we first establish the velocity profiles in the oil drop and water lubrication film, and further provide an expression for the velocity $U_{\text {int }}$ at the o/w interface, in the frame of the capillary tube. We use the notations introduced in Fig. 7. Because the problem is axisymmetric, the velocity $u(r)$ at a given position $\mathrm{z}$ along the $\mathrm{z}$-axis is given by:

For $0<r<r_{\text {cap }}-h$

$$
u(r)=a_{\mathrm{o}} r^{2}+b_{\mathrm{o}}
$$


For $r_{\text {cap }}-h<r<r_{\text {cap }}$

$$
u(r)=a_{w}\left(r_{\text {cap }}-r\right)^{2}
$$

where the indexes o and $\mathrm{w}$ refer respectively to oil and water, and $a_{o}, b_{o}$ and $a_{w}$ are constants. The velocity satisfies the no-slip boundary condition at the capillary wall, $u\left(r_{\text {cap }}\right)=0$.

From continuity of the velocities, one obtains:

$$
a_{w}=\frac{b_{\mathrm{o}}+a_{\mathrm{o}} h^{2}-2 a_{\mathrm{o}} h r_{\mathrm{cap}}+a_{\mathrm{o}} r_{\mathrm{cap}}^{2}}{h^{2}}
$$

The water and oil flowrates are therefore respectively given by:

$$
\begin{aligned}
& Q_{w}=\frac{2}{3}\left(b_{\mathrm{o}} \pi r_{\text {cap }}+a_{\mathrm{o}} \pi r_{\text {cap }}^{3}\right) h+O(h)^{2} \\
& Q_{o}=b_{\mathrm{o}} \pi\left(h-r_{\text {cap }}\right)^{2}+\frac{1}{2} a_{o} \pi\left(h-r_{\text {cap }}\right)^{4}
\end{aligned}
$$

As a result, $Q_{w} / Q_{0} \propto h / r_{\text {cap }}$ and the water flowrate is negligible compared to the oil flowrate. In the following we neglect its contribution to the flowrate, i.e. we consider $Q \approx Q_{o}$.

Stress continuity at the interface yields:

$$
\begin{aligned}
& \left.\left.\eta_{w} \frac{\partial u}{\partial r}\right]_{\left(r_{c a p}-h\right)^{-}}=\eta_{o} \frac{\partial u}{\partial r}\right]_{\left(r_{c a p}-h\right)^{+}}+\frac{\partial \gamma}{\partial z} \\
& -2 a_{w} h \eta_{\mathrm{w}}=\frac{\partial \gamma}{\partial z}+2 a_{o}\left(\mathrm{r}_{\text {cap }}-h\right) \eta_{\mathrm{o}}
\end{aligned}
$$

Using eq. (A2) to (A7) and expanding in powers of $h / r_{\text {cap }}$, one obtains:

$$
U_{\text {int }}=u\left(r_{\text {cap }}-h\right)=-\frac{1}{2 \eta_{\mathrm{w}}} \frac{\partial \gamma}{\partial z} h+\frac{2 Q \eta_{\mathrm{o}}}{\pi r_{\text {cap }}^{3} \eta_{\mathrm{w}}} h+O(h)^{2}
$$

The first term of the right hand term is the Marangoni velocity. The amplitude of the Marangoni effect is measured by the Marangoni number defined as the ratio of the Marangoni and front velocity of the drop, yielding:

$$
M a=\frac{\partial \gamma}{\partial z} \frac{r_{c a p}}{\eta_{\mathrm{w}}} \frac{1}{U_{F}^{1 / 3} U_{c}^{2 / 3}}
$$

Where, for the sake of simplicity, we have used the expression established by Bretherton for the thickness of the lubrication film, $h=r_{c a p} \mathrm{Ca}^{2 / 3}$, rather than eq. (7) and differing with eq. (7) by less than $15 \%$ for the largest capillary number. The capillary number is expressed as $C a=U_{F} / U_{c}$ with $U_{c}=\gamma / \eta_{\mathrm{w}}$ the capillary velocity.

If $L$ is the length over which the interfacial tension gradient extends, $\frac{\partial \gamma}{\partial z} \approx\left(\gamma_{o w}-\gamma_{c m c}\right) / L$. With $L=$ $500 \mu \mathrm{m}$ and $U_{F}$ ranging from $10^{-3}$ to $1 \mathrm{~m} . s^{-1}$, we obtain Marangoni numbers ranging from 0.1 to 1 . 
Interfacial viscosity effects have been neglected. For a surface viscosity $\eta_{S}$, they are measured by the Boussinesq number that compares the surface stress $\sim \eta_{\mathrm{s}} U_{\text {int }} / \rho_{F}\left(z_{F}-z_{B}\right)$ with the viscous stress in the lubrication film $\sim \eta_{\mathrm{w}} U_{\text {int }} / h$ and is therefore given by:

$$
B o=\frac{\eta_{s} h}{\eta_{\mathrm{w}} \rho_{F}\left(z_{F}-z_{B}\right)}
$$

With $\eta_{s}=4.5 \times 10^{-5} \mathrm{~Pa}$. m.s ${ }^{27}$, the maximum value of $B o$ is $10^{-2}$ in our experiments, confirming that the interfacial viscous stress is negligible.

\section{Appendix B: surfactant expansion rate}

Here we compute the surface expansion rate at the front of the drop, as it passes through the contraction. Its general expression is the following:

$$
\dot{A}=\frac{1}{S_{F}} \frac{d S_{F}}{d t}
$$

where $S_{F}$ is the surface of the front spherical cap given by:

$$
S_{F}=\int_{0}^{\frac{\pi}{2}-\alpha} 2 \pi \rho_{F}^{2} \sin \theta d \theta=2 \pi \rho_{F}^{2}\left(\frac{1-\sin \alpha}{\cos ^{2} \alpha}\right)
$$

When the front of the drop moves forward over a distance $d z=z_{F}(t+d t)-z_{F}(t)$, its surface increase results from two contributions, as represented in Fig. 13. The first one corresponds to the expansion of the front spherical cap (red dotted line in Fig. 13), and the second one, to the supplementary surface spreading along the capillary wall (green line). The total surface variation is therefore given by:

$$
d S_{F}=d z \frac{\partial}{\partial z}\left(2 \pi \rho_{F}^{2}(z) \frac{1-\sin \alpha(z)}{\cos ^{2} \alpha(z)}\right)+2 \pi \rho_{F}(z) \frac{d z-U_{z} \frac{d z}{U_{F}}}{\cos \alpha(z)}
$$

Where $\alpha$ is the angle between the tangent to the drop surface at position $z_{F}$ and the horizontal axis.

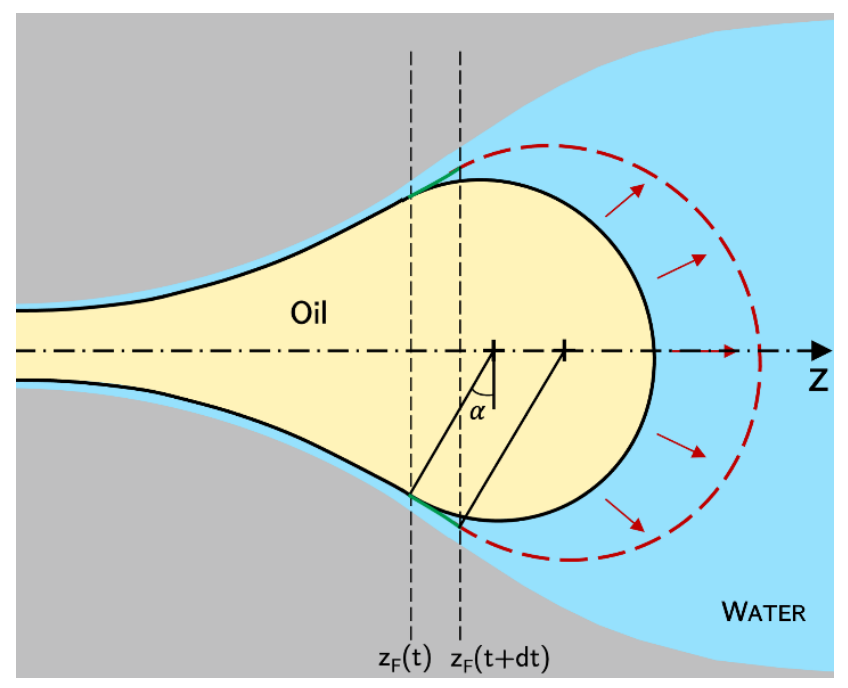

Fig. 13: Schematic representation of the drop at two successive instants in the constriction showing how it surface expands. 
Since $U_{\text {int }}<U_{F}$, the surface expansion rate can be approximated as:

$$
\dot{A}=\frac{\frac{\partial}{\partial z}\left(2 \pi \rho_{F}^{2}(z) \frac{1-\sin \alpha(z)}{\cos ^{2} \alpha(z)}\right)+\frac{2 \pi \rho_{F}(z)}{\cos \alpha}}{2 \pi \rho_{F}^{2}(z) \frac{1-\sin \alpha(z)}{\cos ^{2} \alpha(z)}} U_{F}
$$

In practice, all geometrical parameters in eq. (B4) can be measured by image analysis, as well as the drop front velocity $U_{F}$, allowing measurements of the surface expansion rate at the drop front. The resulting expansion rates measured at the center of the contraction are shown in Fig. 14 for surfactant-laden drops and at different concentrations of surfactants in the aqueous solution.

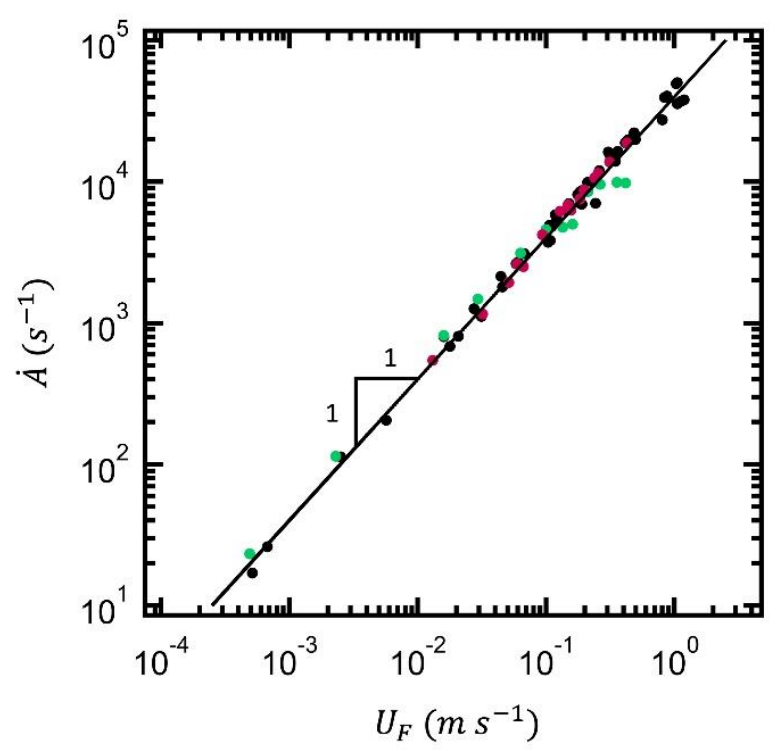

Fig. 14: Surface expansion rate of surfactant-laden droplets as a function of their front velocity, computed following eq. (B4) for surfactant concentrations of $1 \mathrm{cmc}$ (green symbols), $5 \mathrm{cmc}$ (black symbols) and $10 \mathrm{cmc}$ (red symbols). Full line represents eq. (B5) with $\rho_{F}=25 \mu \mathrm{m}$.

The expansion rate increases linearly with the front velocity of the drop, as a result of the negligible derivative term in eq. (B4). Since at the center of the contraction $\alpha=0$, we have found the expansion rate at that point is well described by the simple expression (shown in full line in Fig. 14):

$$
\dot{A}=\frac{U_{F}}{\rho_{F}}
$$

\section{Appendix C: Critical expansion rates for a transfer limited by micelle diffusion or surfactant adsorption}

First, we show here that, if the transfer of surfactants from the bulk to the surface was limited by the diffusion process, then the variations of surface concentration with the expansion rate would depend on the surfactant concentration. A similar calculation is reported in the literature but for concentrations below the $\mathrm{cmc}^{28}$. Here, we consider concentrations that are larger than the $\mathrm{cmc}$; it is therefore the advection/diffusion of micelles that can limit the transfer to the interface since the concentration of free surfactant remains constant. In a 1D description, in the frame of the interface, the equation verified by the surfactants aggregated in micelles, of concentration $c_{M}=c-c_{c m c}$ is : 


$$
u_{z} \frac{\partial c_{M}}{\partial z}+D_{M} \frac{\partial^{2} c_{M}}{\partial z^{2}}=0
$$

Where $u_{z}$ is the velocity of water, and hence of the micelles in the frame of the interface and $D_{M}$ their diffusion coefficient. Note that in this frame $u_{z}=-\dot{A z}$. The solution to eq. (C1) is (see ${ }^{29}$ for the complete demonstration):

$$
c_{M}(z)=C \int_{0}^{z} e^{-\dot{A} y^{2} / 2 D_{M}} d y+B=c_{S}+\frac{c_{\infty}-c_{S}}{\int_{0}^{\infty} e^{-\dot{A} y^{2} / 2 D_{M} d y}} \int_{0}^{z} e^{-\dot{A} y^{2} / 2 D_{M}} d y
$$

Where $c_{\infty}$ and $c_{s}$ are respectively the surfactants- in - micelle concentration far away from the drop and at its interface. The flux of micelles to the drop surface is therefore:

$$
J=D_{M} \frac{\partial c_{M}}{\partial z}=D_{M} \frac{c_{\infty}-c_{S}}{\int_{0}^{\infty} e^{-\dot{A} y^{2} / 2 D_{M} d y}}=D_{M}\left(c_{\infty}-c_{S}\right) 2 \sqrt{\frac{\dot{A}}{2 \pi D_{M}}}
$$

In stationary conditions, the flux balances the decrease rate in surfactant induced by surface expansion $\Gamma_{F} \dot{A}$, yielding:

$$
c_{\infty}-c_{S}=\Gamma_{F} \sqrt{\frac{\pi \dot{A}}{2 D_{m}}}
$$

As long as $c_{S}$ remains positive, there are micelles in the vicinity of the interface, and the interfacial tension remains almost constant. The critical value of the expansion rate above which $\Gamma_{F}$ is expected to decrease corresponds thus to zero micelle concentration close to the surface, i.e. $c_{S}=0$, therefore:

$$
\dot{A_{c}}=\frac{2 D_{m}}{\pi}\left(\frac{c-c_{c m c}}{\Gamma_{c m c}}\right)^{2}
$$

With $D_{m}=10^{-9} \mathrm{~m}^{2} \cdot \mathrm{s}^{-1}$, the critical value of the expansion rate is $\dot{A_{c}}=1.3 \times 10^{3} \mathrm{~s}^{-1}$ for $c=5 c_{c m c}$ and $\dot{A_{c}}=6.7 \times 10^{3} \mathrm{~s}^{-1}$ for $c=10 c_{c m c}$.

Second, we show that if the transfer of surfactants from the bulk to the surface was limited by surfactant adsorption, it would also depend on surfactant concentration.

The expansion of the drop surface decreases the surfactant concentration at the front, $\Gamma_{F}$, below its initial value $\Gamma_{c m c}$ whereas the adsorption from the bulk within a finite adsorption time $\tau$ keeps opposing this decrease. In this picture, the rate of variation of the surface concentration is given by:

$$
\frac{\mathrm{d} \Gamma_{F}}{\mathrm{dt}}=-\dot{A} \Gamma_{F}+\frac{\Gamma_{c m c}-\Gamma_{F}}{\tau}
$$

Assuming a stationary state is reached yields the following expression for the surface concentration:

$$
\Gamma_{F}=\frac{\Gamma_{c m c}}{1+\dot{A} \tau}
$$


According to eq. (C7), the threshold value of the expansion rate above which $\Gamma_{F}$ is expected to decrease is the inverse of the adsorption time, $1 / \tau$. Although not accurately measured, this time has been shown to depend on both concentration and salt content ${ }^{26}$.

\section{References}

1. Zinchenko, A. Z.; Davis, R. H., Motion of Deformable Drops Through Porous Media. Annual Review of Fluid Mechanics 2017, 49, 71-90.

2. Chio, H.; Jensen, M. J.; Wang, X. L.; Bruus, H.; Attinger, D., Transient pressure drops of gas bubbles passing through liquid-filled microchannel contractions: an experimental study. Journal of Micromechanics and Microengineering 2006, 16 (1), 143-149.

3. Cobos, S.; Carvalho, M. S.; Alvarado, V., Flow of oil-water emulsions through a constricted capillary. International Journal of Multiphase Flow 2009, 35 (6), 507-515.

4. Harvie, D. J. E.; Davidson, M. R.; Cooper-White, J. J.; Rudman, M., A parametric study of droplet deformation through a microfluidic contraction: Low viscosity Newtonian droplets. Chemical Engineering Science 2006, 61 (15), 5149-5158.

5. Tsai, T. M.; Miksis, M. J., DYNAMICS OF A DROP IN A CONSTRICTED CAPILLARY-TUBE. Journal of Fluid Mechanics 1994, 274, 197-217.

6. Jensen, M. J.; Goranovic, G.; Bruus, H., The clogging pressure of bubbles in hydrophilic microchannel contractions. Journal of Micromechanics and Microengineering 2004, 14 (7), 876-883.

7. Singla, A.; Ray, B., Effects of surface topography on low Reynolds number droplet/bubble flow through a constricted passage. Physics of Fluids 2021, 33 (1).

8. Graham, D. R.; Higdon, J. J. L., Oscillatory flow of droplets in capillary tubes. Part 2. Constricted tubes. Journal of Fluid Mechanics 2000, 425, 55-77.

9. Jang, J. B.; Sun, Z. H.; Santamarina, J. C., Capillary pressure across a pore throat in the presence of surfactants. Water Resources Research 2016, 52 (12), 9586-9599.

10. Tsai, T. M.; Miksis, M. J., The effects of surfactant on the dynamics of bubble snap-off. Journal of Fluid Mechanics 1997, 337, 381-410.

11. Gissinger, J. R.; Zinchenko, A. Z.; Davis, R. H., Drops with insoluble surfactant squeezing through interparticle constrictions. Journal of Fluid Mechanics 2019, 878, 324-355.

12. Aveyard, R.; Clint, J. H.; Nees, D.; Quirke, N., Structure and collapse of particle monolayers under lateral pressure at the octane/aqueous surfactant solution interface. Langmuir 2000, 16 (23), 8820-8828.

13. Xu, H.; Melle, S.; Golemanov, K.; Fuller, G., Shape and buckling transitions in solid-stabilized drops. Langmuir 2005, 21 (22), 10016-10020.

14. Asekomhe, S. O.; Chiang, R.; Masliyah, J. H.; Elliott, J. A. W., Some observations on the contraction behavior of a water-in-oil drop with attached solids. Industrial \& Engineering Chemistry Research 2005, 44 (5), 1241-1249.

15. Samudrala, N.; Nam, J.; Sarfati, R.; Style, R. W.; Dufresne, E. R., Mechanical stability of particle-stabilized droplets under micropipette aspiration. Physical Review E 2017, 95 (1).

16. Garbin, V., Collapse mechanisms and extreme deformation of particle-laden interfaces. Current Opinion in Colloid \& Interface Science 2019, 39, 202-211.

17. Gu, C. A.; Botto, L., Buckling vs. particle desorption in a particle-covered drop subject to compressive surface stresses: a simulation study. Soft Matter 2018, 14 (5), 711-724.

18. Jousse, F.; Lian, G. P.; Janes, R.; Melrose, J., Compact model for multi-phase liquid-liquid flows in microfluidic devices. Lab on a Chip 2005, 5 (6), 646-656.

19. Moulik, S. P.; Haque, M. E.; Jana, P. K.; Das, A. R., Micellar properties of cationic surfactants in pure and mixed states. Journal of Physical Chemistry 1996, 100 (2), 701-708.

20. Zdziennicka, A.; Szymczyk, K.; Krawczyk, J.; Janczuk, B., Activity and thermodynamic parameters of some surfactants adsorption at the water-air interface. Fluid Phase Equilibria 2012, 318, 25-33.

21. Kedar, V.; Bhagwat, S. S., Effect of salinity on the IFT between aqueous surfactant solution and crude oil. Petroleum Science and Technology 2018, 36 (12), 835-842. 
22. Frelichowska, J.; Bolzinger, M. A.; Chevalier, Y., Effects of solid particle content on properties of o/w Pickering emulsions. Journal of Colloid and Interface Science 2010, 351 (2), 348-356.

23. Bretherton, F. P., THE MOTION OF LONG BUBBLES IN TUBES. Journal of Fluid Mechanics 1961, 10 (2), 166188.

24. Aussillous, P.; Quere, D., Quick deposition of a fluid on the wall of a tube. Physics of Fluids 2000, 12 (10), 2367-2371.

25. Klaseboer, E.; Gupta, R.; Manica, R., An extended Bretherton model for long Taylor bubbles at moderate capillary numbers. Physics of Fluids 2014, 26 (3).

26. Petkova, B.; Tcholakova, S.; Chenkova, M.; Golemanov, K.; Denkov, N.; Thorley, D.; Stoyanov, S., Foamability of aqueous solutions: Role of surfactant type and concentration. Advances in Colloid and Interface Science 2020, 276.

27. Huhnerfuss, H., SURFACE VISCOSITY MEASUREMENTS - A REVIVAL OF A NEARLY FORGOTTEN SURFACE CHEMICAL METHOD. Journal of Colloid and Interface Science 1985, 107 (1), 84-95.

28. Christov, N. C.; Danov, K. D.; Kralchevsky, P. A.; Ananthapadmanabhan, K. P.; Lips, A., Maximum bubble pressure method: Universal surface age and transport mechanisms in surfactant solutions. Langmuir 2006, 22 (18), 7528-7542.

29. Levich, V. G., Physicochemiral Hydrodynamics. Prentice-Hall, Inc: New-Jersey, 1962. 